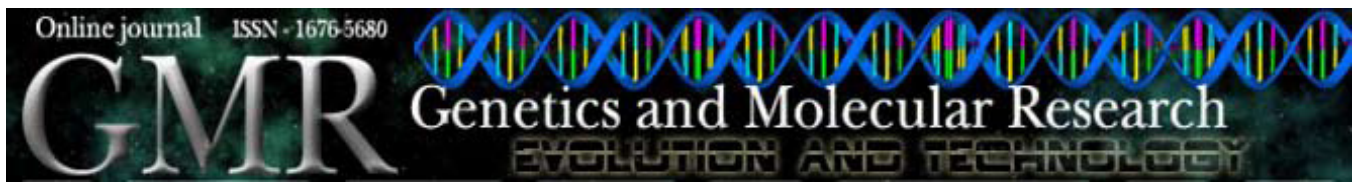

$\underline{\text { Viewpoint }}$

\title{
Phylogenetic analysis of oryx species using partial sequences of mitochondrial rRNA genes
}

\author{
H.A. Khan, I.A. Arif, A.H. Al Farhan and A.A. Al Homaidan \\ Molecular Fingerprinting and Biodiversity Unit, \\ Prince Sultan Research Chair Program in Environment and Wildlife, \\ College of Science, King Saud University, Riyadh, Saudi Arabia \\ Corresponding author: H.A. Khan \\ E-mail: khan_haseeb@yahoo.com
}

Genet. Mol. Res. 7 (4): 1150-1155 (2008)

Received July 2, 2008

Accepted August 6, 2008

Published October 28, 2008

\begin{abstract}
We conducted a comparative evaluation of $12 \mathrm{~S}$ rRNA and 16S rRNA genes of the mitochondrial genome for molecular differentiation among three oryx species (Oryx leucoryx, Oryx dammah and Oryx gazella) with respect to two closely related outgroups, addax and roan. Our findings showed the failure of $12 \mathrm{~S}$ rRNA gene to differentiate between the genus Oryx and addax, whereas a 342-bp partial sequence of $16 \mathrm{~S}$ rRNA accurately grouped all five taxa studied, suggesting the utility of $16 \mathrm{~S}$ rRNA segment for molecular phylogeny of oryx at the genus and possibly species levels.
\end{abstract}

Key words: Mitochondrial DNA; rRNA genes; Phylogeny; Endangered animals; Oryx species 
Information on the genetic diversity of wildlife is necessary to identify genetically deteriorated populations and to establish better management plans for the conservation and recovery of endangered populations (Frankham et al., 2002; Russello and Amato, 2007). An accurate molecular classification of endangered species strengthens our understanding about the diversity and identification of distinct populations to be managed carefully. It is well recognized that inbreeding affects the fitness in captive animals due to inbreeding depression in wild populations (Crnokrak and Roff, 1999). Wildlife populations with lower genetic diversity are at greater risk of extinction (Saccheri et al., 1998) and such endangered populations often fail to exhibit signs of recovery until crossed with individuals from other populations (Westemeier et al., 1998; Land and Lacy, 2000). Although, distantly related individuals are used for maximizing outbreeding, closely related individuals need to be introduced to recipient populations for maintaining the genetic identity of a population. Thus, knowledge of the relatedness between individuals is particularly important in captive breeding programs to monitor the impacts of inbreeding or outbreeding on genetic diversity (Montgomery et al., 1998; Frankham et al., 2002).

Molecular markers play an important role in estimating the relatedness among various taxa. Mitochondrial DNA (mtDNA) is regarded as an important tool in studying the evolutionary relationship among various species due to its conservativeness in protein-coding regions, high variability in non-coding sequences and lack of recombination (Olivo et al., 1983; Ingman et al., 2000). Sequence divergence accumulates more rapidly in mtDNA than nuclear DNA due to faster mutation rates and lack of a repair system in mtDNA, often rendering it with high levels of informative variation. Of greater utility are the two ribosomal RNA genes (12S rRNA and 16S rRNA), a few coding genes (e.g., cytochrome b, ATPase and NADH dehydrogenase) and control region sequences.

The Arabian oryx (Figure 1) formerly inhabited all of the Arabian Peninsula but was extirpated from the wild in the early 1970s (Henderson, 1974), and it was first reintroduced in Oman in 1982 (Spalton et al., 1999). In Saudi Arabia, the Arabian oryx was first released in 1990 into a protected desert area in the west-central region (Ostrowski et al., 1998) and later reintroduced into a protected sand dune area (Mesóchina et al., 2003). Mesóchina and colleagues (2003) have built up a captive oryx population recognized as the most polymorphic of all captive herds, suggesting that no recent management-related bottleneck has occurred. However, genetic analysis has suggested that perhaps as much as $50 \%$ of the neutral genetic variation present in the pre-extinction population of Arabian oryx is absent from contemporary populations (Marshall et al., 1999). Iyengar et al. (2007) have recommended the maintenance of a global perspective for the genetic management of captive oryx, and that individuals from various management programs and regions need to be effectively utilized for sustained future captive breeding in order to ensure that the vital remnants of genetic diversity are retained and represented in future reintroduction programs. Recently, mtDNA control region and cytochrome b sequence data have been used to determine the genetic diversity and phylogenetic relationship in oryx species (Masembe et al., 2006; Iyengar et al., 2006). In this investigation, we performed a comparative evaluation of $12 \mathrm{~S}$ rRNA and $16 \mathrm{~S}$ rRNA genes for their utilization in the understanding of the molecular phylogeny of oryx species. 

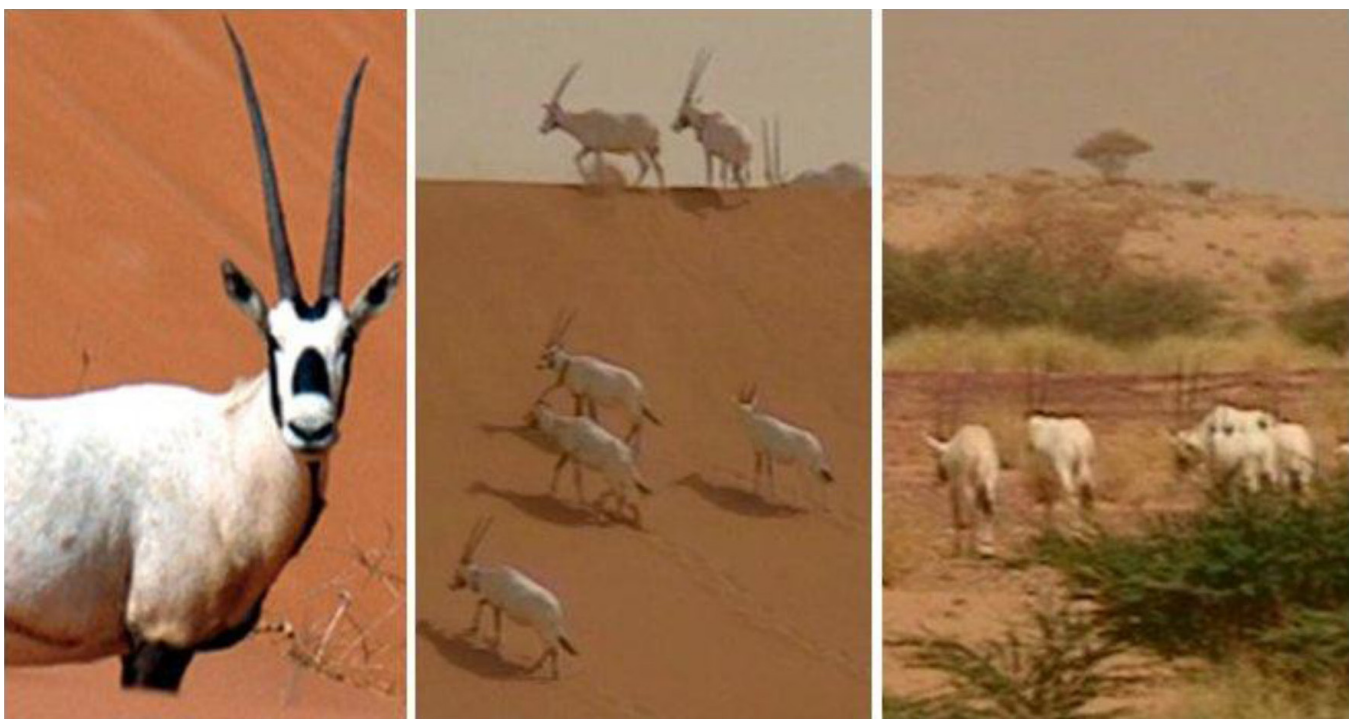

Figure 1. Arabian oryx (Oryx leucoryx): an endangered species.

The partial sequences of 12S rRNA and 16S rRNA genes of the three oryx species including Oryx leucoryx, Oryx dammah and Oryx gazella were obtained from GenBank. Two closely related taxa including addax (Addax nasomaculatus) and roan (Hippotragus equines), which differ from oryx at the genus level, were used as outgroups. The GenBank accession numbers for 12S rRNA and 16S rRNA of the five taxa analyzed are: Oryx leucoryx (U86971; U87021), Oryx dammah (U86970; U87020), Oryx gazella (U86972; U87022), addax (U86973; U87023), and roan (U86975; U87025), respectively. There were a total of 241 and 342 positions in the partial sequence datasets of $12 \mathrm{~S}$ rRNA and $16 \mathrm{~S}$ rRNA. The sequences were first examined for alignment using the BIOEDIT software (Hall, 1999). Estimates of average evolutionary divergence over all sequence pairs were computed by averaging the number of base substitutions per site. Phylogenetic analyses were performed using the evolutionary distances computed by the maximum composite likelihood method in MEGA 4 (Tamura et al., 2004, 2007). The evolutionary history was inferred using the unweighted pair group method with arithmetic mean (Sneath and Sokal, 1973). The original tree provides confidence levels for each clade, whereas the bootstrap consensus tree collapses the weakest clades. The bootstrap consensus trees inferred from 1000 replicates (Felsenstein, 1985) were taken to represent the evolutionary history of the taxa analyzed.

Both the frequency of polymorphic sites and the average evolutionary divergence were found to be comparatively less in $12 \mathrm{~S}$ rRNA than in $16 \mathrm{~S}$ rRNA. The results of phylogenetic analysis clearly showed the failure of $12 \mathrm{~S}$ rRNA to differentiate between the genus Oryx and addax (Figure 2). However, both original and bootstrap consensus trees for 16S rRNA efficiently discriminated among various genera as well as oryx species (Figure 2). 


\section{A. 12S rRNA}
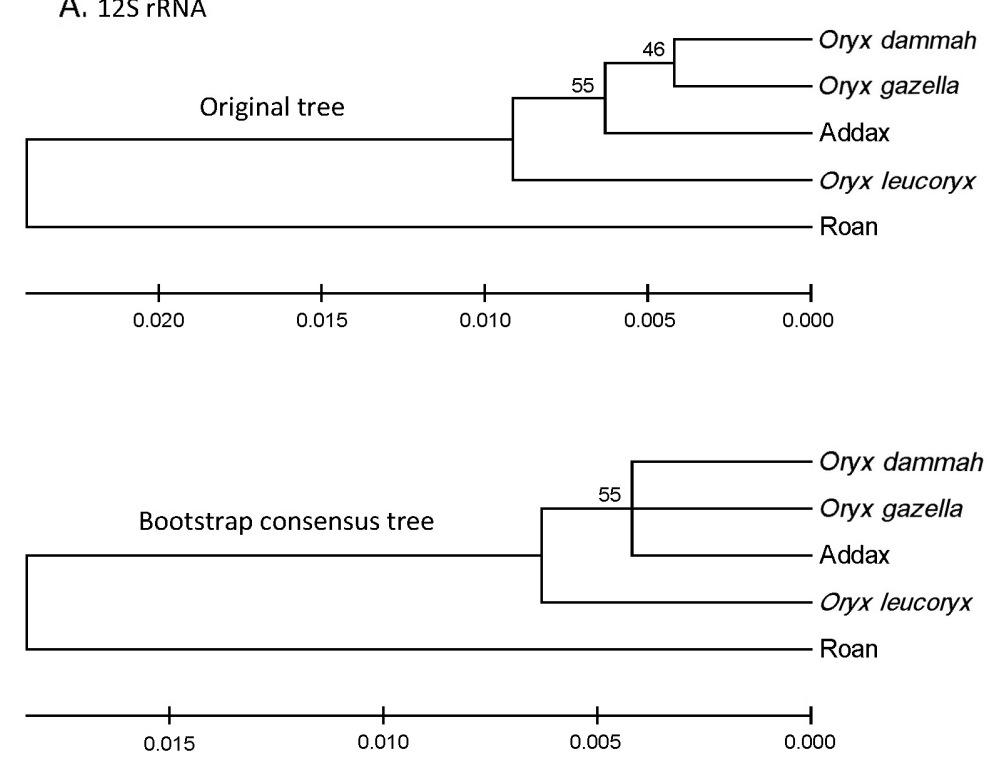

B. 165 rRNA
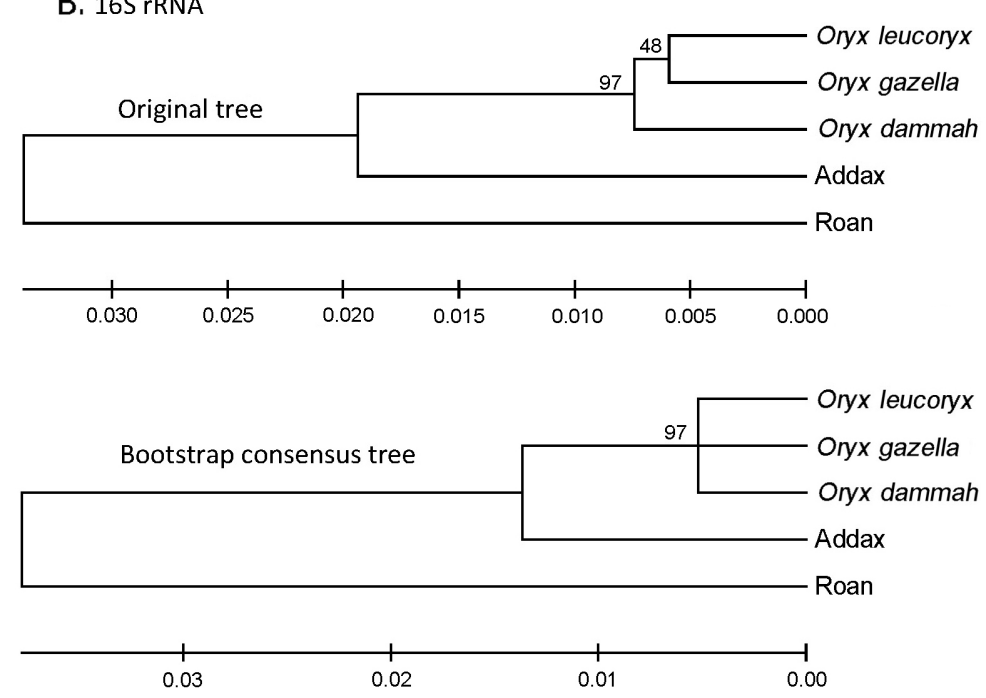

Figure 2. Original and bootstrap consensus trees for 12S rRNA and 16S rRNA genes. The percentage of replicate trees in which the associated taxa clustered together in the bootstrap test (1000 replicates) is shown next to the branches. Branches corresponding to partitions reproduced in less than $50 \%$ bootstrap replicates are collapsed in bootstrap consensus trees. 
The molecular phylogeny with $16 \mathrm{~S}$ rRNA sequences demonstrated that Oryx leucoryx is grouped with Oryx gazella rather than Oryx dammah, though with a low bootstrap support. These findings are identical to an earlier report based on using approximately 1250-bp sequences of the mitochondrial control region of oryx species (Iyengar et al., 2006). Although mtDNA evolves faster than the nuclear genome, the rate of evolution is different for different regions of mtDNA and has been used to examine various phylogenetic relationships. Since the $12 \mathrm{~S}$ rRNA gene is highly conserved, it therefore failed to accurately differentiate taxa at the genus level but could be applied to illustrate phylogeny of higher categorical levels such as in phyla or subphyla. The 16S rRNA gene is regarded as a highly useful segment of the mitochondrial genome for phylogenetic studies at midcategorical levels such as families or genera (Gerber et al., 2001). In conclusion, this study demonstrates the ability of the 16S rRNA gene for differentiation of oryx species from other closely related taxa.

\section{REFERENCES}

Crnokrak P and Roff DA (1999). Inbreeding depression in the wild. Heredity 83 (Pt 3): 260-270.

Felsenstein J (1985). Confidence limits on phylogenies: an approach using the bootstrap. Evolution 39: 783-791.

Frankham R, Ballou JD and Briscoe DA (2002). Introduction to Conservation Genetics. Cambridge University Press, New York.

Gerber AS, Loggins R, Kumar S and Dowling TE (2001). Does nonneutral evolution shape observed patterns of DNA variation in animal mitochondrial genomes? Annu. Rev. Genet. 35: 539-566.

Hall TA (1999). BioEdit: a user-friendly biological sequence alignment editor and analysis program for Windows 95/98/ NT. Nucleic Acids Symp. Ser. 41: 95-98.

Henderson DS (1974). Were they the last Arabian oryx? Oryx 12: 347-350.

Ingman M, Kaessmann H, Paabo S and Gyllensten U (2000). Mitochondrial genome variation and the origin of modern humans. Nature 408: 708-713.

Iyengar A, Diniz FM, Gilbert T, Woodfine T, et al. (2006). Structure and evolution of the mitochondrial control region in oryx. Mol. Phylogenet. Evol. 40: 305-314.

Iyengar A, Gilbert T, Woodfine T, Knowles JM, et al. (2007). Remnants of ancient genetic diversity preserved within captive groups of scimitar-horned oryx (Oryx dammah). Mol. Ecol. 16: 2436-2449.

Land ED and Lacy RC (2000). Introgression level achieved through Florida Panther genetic restoration. Endanger. Spec. Update 17: 99-103.

Marshall TC, Sunnocks P, Spalton JA, Greth A, et al. (1999). Use of genetic data for conservation management: the case of the Arabian oryx. Anim. Conserv. 2: 269-278.

Masembe C, Muwanika VB, Nyakaana S, Arctander P, et al. (2006). Three genetically divergent lineages of the Oryx in eastern Africa: Evidence for an ancient introgressive hybridization. Conserv. Genet. 7: 551-562.

Mesóchina P, Bedin E and Ostrowski S (2003). Reintroducing antelopes into arid areas: lessons learnt from the oryx in Saudi Arabia. C. R. Biol. 326 (Suppl. 1): S158-S165.

Montgomery ME, Ballou JD, Nurthen RK, England PR, et al. (1998). Minimizing kinship in captive breeding programs. Zoo Biol. 16: 377-389.

Olivo PD, Van de Walle MJ, Laipis PJ and Hauswirth WW (1983). Nucleotide sequence evidence for rapid genotypic shifts in the bovine mitochondrial DNA D-loop. Nature 306: 400-402.

Ostrowski S, Bedin E, Lenain D and Abuzinada AH (1998). Ten years of Arabian oryx conservation breeding in Saudi Arabia - achievements and regional perspectives. Oryx 32: 209-222.

Russello MA and Amato G (2007). On the horns of a dilemma: molecular approaches refine ex situ conservation in crisis. Mol. Ecol. 16: 2405-2406.

Saccheri I, Kuussaari M, Kankare M, Vikman P, et al. (1998). Inbreeding and extinction in a butterfly metapopulation. Nature 392: 491-494.

Sneath PHA and Sokal RR (1973). Numerical Taxonomy. Freeman, San Francisco.

Spalton JA, Lawrence MW and Brend SA (1999). Arabian oryx reintroduction in Oman: successes and setbacks. Oryx 33: $168-175$. 
Tamura K, Nei M and Kumar S (2004). Prospects for inferring very large phylogenies by using the neighbor-joining method. Proc. Natl. Acad. Sci. U. S. A. 101: 11030-11035.

Tamura K, Dudley J, Nei M and Kumar S (2007). MEGA4: Molecular evolutionary genetics analysis (MEGA) software version 4.0. Mol. Biol. Evol. 24: 1596-1599.

Westemeier RL, Brawn JD, Simpson SA, Esker TL, et al. (1998). Tracking the long-term decline and recovery of an isolated population. Science 282: 1695-1698. 